\title{
Systematically Peculiar Molecular Composition in M 82: Regarding the Formation Mechanisms
}

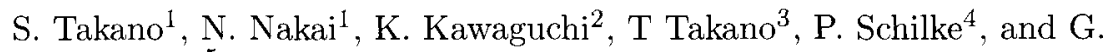 \\ Winnewisser ${ }^{5}$ \\ ${ }^{1}$ Nobeyama Radio Observatory, National Astronomical Observatory of \\ Japan, Nobeyama, Minamimaki, Minamisaku, 384-1305 Japan \\ ${ }^{2}$ Department of Chemistry, Okayama University, Tsushima-naka 3-1-1, \\ Okayama, 700-8530 Japan \\ ${ }^{3}$ Graduate School of Science and Technology, Chiba University, \\ Yayoi-cho 1-33, Inage-ku, Chiba, 263-8522 Japan \\ ${ }^{4}$ MPIfR, Auf dem Hügel 69, D-53121 Bonn, Germany \\ ${ }^{5}$ I. Physikalisches Institut, Universität zu Köln, Zülpicher Strasse 77, \\ D-50937 Köln, Germany
}

\begin{abstract}
A systematically peculiar molecular composition has been found in a nearby starburst galaxy M 82 . Molecules related to grain surface formation and to production reactions favorable at high-temperature are deficient in M 82 among nearby galaxies with rich gas. These molecules are $\mathrm{SO}, \mathrm{SiO}, \mathrm{NH}_{3}, \mathrm{HNCO}$, $\mathrm{CH}_{3} \mathrm{OH}$, and $\mathrm{CH}_{3} \mathrm{CN}$. Possible reasons for this peculiarity are discussed.
\end{abstract}

\section{Introduction}

More than 20 molecules have been detected in external galaxies. Studies of the relation between their abundances and the physical conditions are important to understand physical and chemical processes in external galaxies. Two starburst galaxies, NGC 253 and M 82, are known to be suitable for such study; the $\mathrm{H}_{2}$ column densities are high and nearly identical, and their distances are nearly equal (about $3 \mathrm{Mpc}$ ) each other. Several major molecules have been detected in both of the galaxies with similar abundances (e.g., CO, CS). However, it is known that $\mathrm{SO}, \mathrm{SiO}, \mathrm{NH}_{3}, \mathrm{HNCO}, \mathrm{CH}_{3} \mathrm{OH}$, and $\mathrm{CH}_{3} \mathrm{CN}$ have been clearly detected in NGC 253, but they have not been, or only only barely, detected with comparable sensitivity in M 82. In Table 1 quantitative information concerning the abundances of these molecules in the two galaxies is given. The CS molecule is also listed as one example with similar abundances.

\section{Systematically Peculiar Molecular Composition in M 82}

We found a common characteristic of the above cited six molecules; they are efficiently produced under high-temperature conditions, and/or they are originated from grain by evaporation processes (Takano, Nakai, \& Kawaguchi 1995). 
Table 1. Molecular abundance (relative to $\mathrm{H}_{2}$ ) in NGC 253 and M 82 .

\begin{tabular}{|c|c|c|c|}
\hline & NGC 253 & M 82 & reference \\
\hline $\mathrm{SO}$ & $2 \times 10^{-9}$ & $<7 \times 10^{-10}$ & Takano, Nakai, \& Kawaguchi (1995) \\
\hline $\mathrm{SiO}$ & $8 \times 10^{-10}$ & $<4 \times 10^{-11}$ & Henkel \& Mauersberger (1992) \\
\hline & & $0.4-3.5 \times 10^{-10}$ & García-Burillo et al. (2002) \\
\hline $\mathrm{NH}_{3}$ & $3 \times 10^{-8}$ & $\begin{array}{l}<1 \times 10^{-9} \\
5 \times 10^{-10}\end{array}$ & $\begin{array}{l}\text { Takano, Nakai, \& Kawaguchi (2002) } \\
\text { Weiß et al. (2001) }\end{array}$ \\
\hline $\mathrm{HNCO}$ & $1 \times 10^{-9}$ & $<1 \times 10^{-10}$ & $\begin{array}{l}\text { N-Q-Rieu et al. (1991); } \\
\text { Henkel \& Mauersberger (1992) }\end{array}$ \\
\hline $\mathrm{CH}_{3} \mathrm{OH}$ & $1 \times 10^{-9}$ & $<1 \times 10^{-10}$ & $\begin{array}{l}\text { Hüttemeister, Mauersberger, } \\
\text { \& Henkel (1997) }\end{array}$ \\
\hline $\mathrm{CH}_{3} \mathrm{CN}$ & $5 \times 10^{-10}$ & $<5 \times 10^{-11}$ & Mauersberger et al. (1991) \\
\hline $\mathrm{CS}$ & $1 \times 10^{-9}$ & $1 \times 10^{-9}$ & N-Q-Rieu, Nakai, \& Jackson (1989) \\
\hline
\end{tabular}

The conditions of molecular formation in NGC 253 and M 82 are, therefore, estimated to be rather different.

To study the reason for this difference, and to know which galaxy is peculiar, we also compared the abundances of the above six molecules in other nearby galaxies with rich molecular gas based on our observations and on literature values. As a result, at least $\mathrm{NH}_{3}, \mathrm{HNCO}$, and/or $\mathrm{CH}_{3} \mathrm{OH}$ are abundant enough to be detected in NGC 6946, IC 342, Maffei 2, M 51, Cen A, and NGC 4945 with comparable sensitivity as shown in Table 2 .

Table 2. Detection/non-detection of the six molecules of interest in nearby galaxies with rich molecular gas ${ }^{1}$.

\begin{tabular}{lcccccc}
\hline \hline Galaxy & $\mathrm{SO}$ & $\mathrm{SiO}$ & $\mathrm{NH}_{3}$ & $\mathrm{HNCO}$ & $\mathrm{CH}_{3} \mathrm{OH}$ & $\mathrm{CH}_{3} \mathrm{CN}$ \\
\hline NGC 253 (all yes) & yes & $\begin{array}{c}\text { yes } \\
\text { no }\end{array}$ & $\begin{array}{c}\text { yarely } \\
\text { barely }\end{array}$ & $\begin{array}{c}\text { yes } \\
\text { no }\end{array}$ & $\begin{array}{c}\text { yes } \\
\text { no }\end{array}$ & $\begin{array}{c}\text { yes } \\
\text { no }\end{array}$ \\
\hline NGC 6946 & no & $\ldots$ & no & (yes) & yes & $\ldots$ \\
IC 342 & (yes) & no & yes & yes & yes & $\ldots$ \\
Maffei 2 & $\ldots$ & no & yes & yes & yes & $\ldots$ \\
Cen A & $\ldots$ & no & yes & yes & no & $\ldots$ \\
NGC 4945 & yes & no & & yes & yes & $\ldots$ \\
\hline \hline
\end{tabular}

1 "yes" means detection, "no" non-detection, "barely" weak detection ( 1 order less than NGC 253), "..." no data. References: see Takano, Nakai, \& Kawaguchi (2002)

Quantitative abundances of ammonia and methanol are shown in Figure 1. We conclude that the molecular composition in M 82 is systematically peculiar regarding the formation mechanisms of molecules. The molecules related to dust 

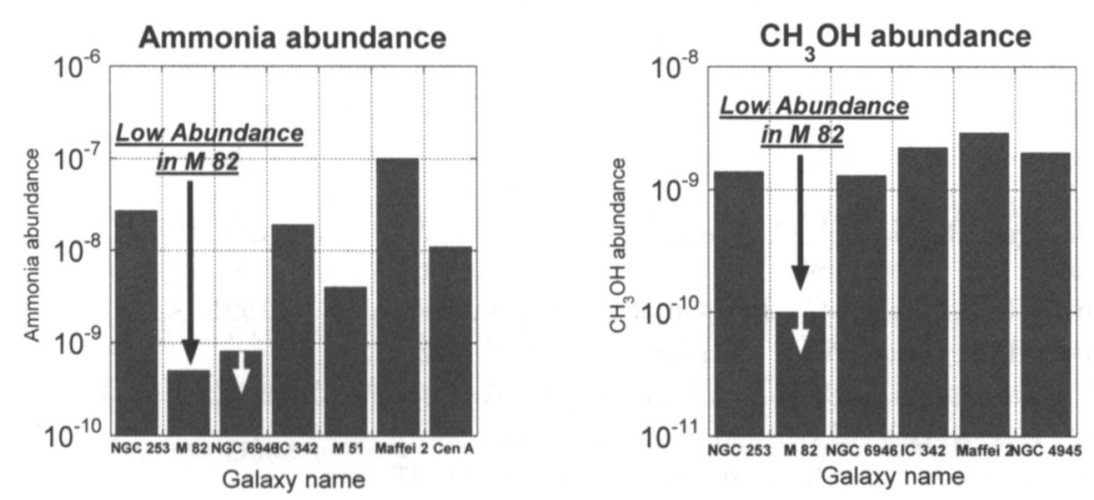

Figure 1. The abundances of ammonia (left) and methanol (right) in nearby galaxies. The white arrows indicate upper limits. The ammonia abundances are taken from Seaquist \& Bell (1990), Takano et al. (2000), Weiß et al. (2001), and Takano et al. (2002). The methanol abundances are taken from Henkel et al. (1990), and Hüttemeister, Mauersberger, \& Henkel (1997).

and efficiently produced under high-temperature conditions are deficient in $M$ 82 among nearby galaxies with rich gas.

\section{Discussion}

As possible reasons for the peculiarity, first, we investigated the amount of dust in these galaxies. According to the IRAS low resolution spectra (LRS), we found that silicate (dust core) is not deficient in M 82. On the other hand, we noticed that Devereux \& Young (1990) reported that the gas-to-warm dust ratios were increased by about a factor of 2 in M 82 compared to those in other nearby galaxies. This result indicates that "warm dust" is somewhat deficient in M 82 , and this may explain to some extent the deficiency of dust related molecules in $\mathrm{M} 82$.

Second, recent estimates of temperatures obtained from ammonia in these galaxies indicate that the rotational temperature at the region with ammonia emission in M 82 is fairly low (29 K and, for example, 100-142 K in NGC 253 reported by Mauersberger et al. 2003; Oike et al. 2004). This result is consistent with the deficiency of dust related molecules in M 82.

It appears that the formation of molecules on dust and/or evaporation to gas-phase is not efficient in M 82. A detailed discussion was given by Takano et al. (2002).

\section{References}

Devereux, N.A. \& Young, J.S. 1990, ApJ, 359, 42

García-Burillo, S., Martín-Pintado, J., Fuente, A., \& Neri, R. 2002, ApJ, 563, L27 
Henkel, C. \& Mauersberger, R. 1992, in IAU Symp. 150, Astrochemistry of Cosmic Phenomena, ed. P. D. Singh (Dordrecht: Kluwer Academic Publishers), 111

Henkel, C., Whiteoak, J.B., Nyman, L.-A., \& Harju, J. 1990, A\&A, 230, L5

Hüttemeister, S., Mauersberger, R., \& Henkel, C. 1997, A\&A, 326, 59

Mauersberger, R., Henkel, C., Walmsley, C.M., Sage, L.J., \& Wiklind, T. 1991, A\&A, 247,307

Mauersberger, R., Henkel, C., Weiß, A., Peck, A.B., \& Hagiwara, Y. 2003, A\&A, 403, 561

Nguyen-Q-Rieu, Henkel, C., Jackson, J.M., \& Mauersberger, R. 1991, A\&A, 241, L33

Nguyen-Q-Rieu, Nakai, N., \& Jackson, J.M. 1989, A\&A, 220, 57

Oike, T., Kawaguchi, K., Takano, S., \& Nakai, N. 2004, PASJ, 56, 431

Seaquist, E.R. \& Bell, M.B. 1990, ApJ, 364, 94

Takano, S., Nakai, N., \& Kawaguchi, K. 1995, PASJ, 47, 801

Takano, S., Nakai, N., \& Kawaguchi, K. 2002, PASJ, 54, 195

Takano, S., Nakai, N., Kawaguchi, K., \& Takano, T. 2000, PASJ, 52, L67

Weiß, A., Neininger, N., Henkel, C., Stutzki, J., \& Klein, U. 2001, ApJ, 554, L143 